\title{
Bilateral saccular aneurysms of the internal carotid artery in the cavernous sinus
}

\author{
CHARLES B. WILSON AND FAY K. MYERS \\ From the Departments of Neurosurgery and Neurology, Louisiana State University School of Medicine, \\ and the Charity Hospital of Louisiana in New Orleans
}

Only 11 cases of unruptured bilateral intracavernous carotid aneurysms have been reported in the literature. Four of these were diagnosed during life. These were reported by Mahoudeau, Daum, George, and Rosier (1949), by Logue (1951), by Alpers, Schlezinger, and Tassman (1951), and by Seltzer and Hurteau (1957). We wish to add an additional case diagnosed during life.

\section{CASE REPORT}

A.A. (No. L-61-382940), a 54-year-old white woman (Fig. 1) was admitted to the Charity Hospital of New Orleans on 10 July 1961. She complained of the sudden onset of inward deviation of the right eye one year before admission and intermittent right retro-orbital headaches for the five months preceding this admission. Positive physical findings were complete paralysis of the external rectus muscle and partial paralysis of the superior and inferior recti muscles of the right eye. A dilated fixed pupil on the same side was present. Visual fields by confrontation were normal. Her corrected visual acuity was $20 / 80$ in the right eye and 20/30 in the left eye. An area of myopic degeneration adjacent to the right papilla was seen. The right corneal reflex was absent and there was tactile hypaesthesia in the distribution of the three divisions of the fifth cranial nerve. The muscles of mastication were paretic on the right side. A systolic bruit was audible over the left eye; none was heard on the right. Proptosis and pulsation of the globe were absent. The electroencephalogram was normal. Skull films showed erosion and widening of the right superior orbital fissure in the frontal projection (Fig. 2). No intrasellar or parasellar calcification was seen. The lateral view of the right carotid arteriogram (Fig. 5) showed a large multilobulated saccular aneurysm in the region of the cavernous sinus and poor filling of the anterior cerebral artery. The internal carotid bifurcation was elevated and the portion of the internal carotid proximal to the bifurcation was sharply angulated. In the frontal projection (Fig. 3), the dome of the aneurysm was situated superiorly and laterally to the carotid bifurcation. The first portion of the middle cerebral artery was stretched over the upper surface of the aneurysm. A smaller intracavernous aneurysm was seen on the left carotid arteriogram (Fig 6). The frontal projection (Fig. 4) was made during contra- lateral carotid compression. There was no deformity of the supra-clinoid segment of the internal carotid artery.

Thirty-six hours following angiography the patient had a series of generalized clonic seizures, with transient postictal flaccid paralysis of the left upper extremity. A persistent bruit over the right globe was detected for the first time. As it was likely that symptoms would be produced from the asymptomatic left aneurysm by ligation of the right carotid artery surgery was ruled out and the patient was discharged. She was last seen on 2 April 1962. She had experienced no recurrence of headaches or seizures. She was receiving anticonvulsant medicationo Additional neurological findings were paresis of elevation of the left eye and increased loss of vision in the right eye Corrected visual acuity of the right eye was 20/200. The cornea was vascularized and the retina could not be visualized. The central visual field in the right eye showed severe peripheral constriction. Visual acuity and fieldso in the left eye were normal.

\section{REVIEW OF THE LITERATURE}

The manner of this patient's presentation was similar to that of the 11 cases reported in the literature. The available information on the cases, including the present case, is listed in Table I. Four patients had bilateral cranial nerve signs. Ten complained of headaches. Bruits were noted on two patients. Radiographs showed abnormalities of the sella, clinoids, or sphenoid bone in eight patients, and unilateral or bilateral calcification in the walls of the aneurysms in four cases. In only two cases was enlargement of the superior orbital fissure mentioned, whereas Rischbieth and Bull (1958) reported significant expansion of this structure in $74 \%$ of 23 cases of infraclinoid aneurysms. Eight patients were dead within 18 months of the initial examination. Of the seven cases on which necropsies were performed, one showed rupture of one aneurysm, which was old. In these cases either death was attributed to extracranial causes or the cause of death was not stated. Bozzoli (1937) reported an intriguing case with active acromegaly, in which the pituitary was histologically normal at necropsy. 


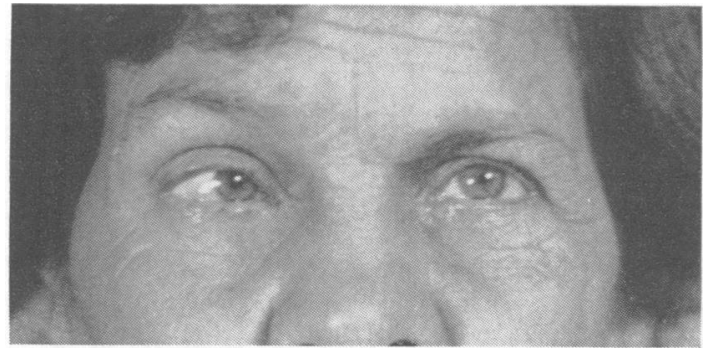

FIG. 1

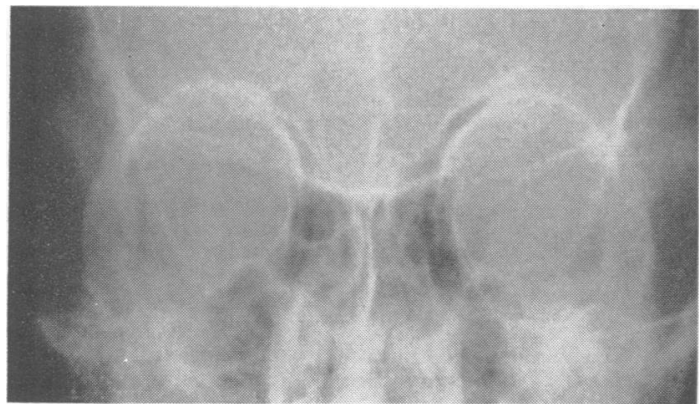

FIG. 2

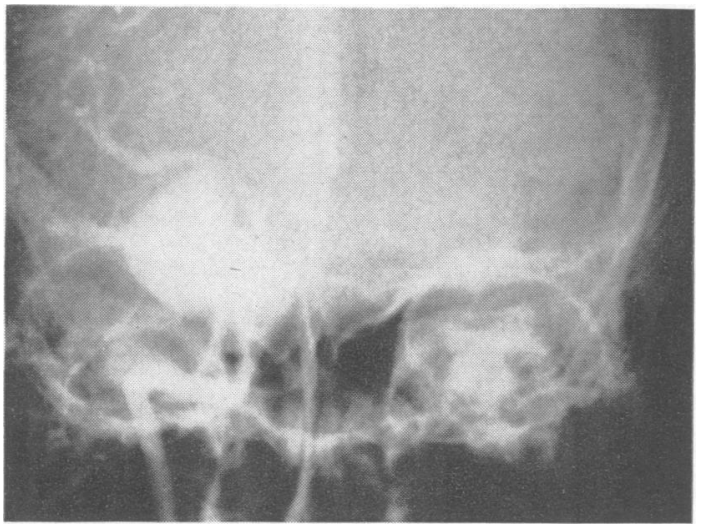

FIG. 3

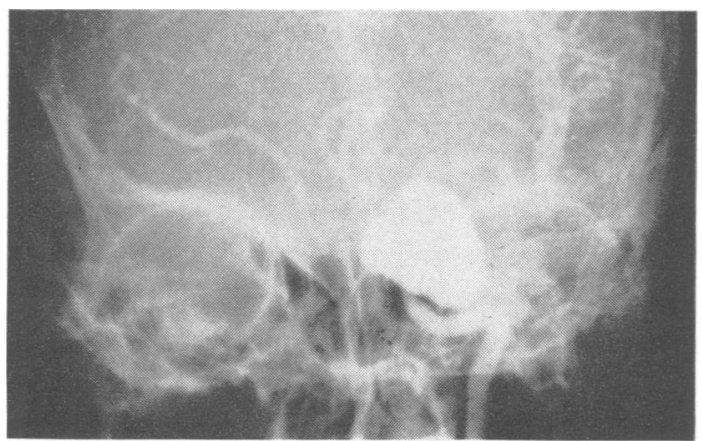

FIG. 4

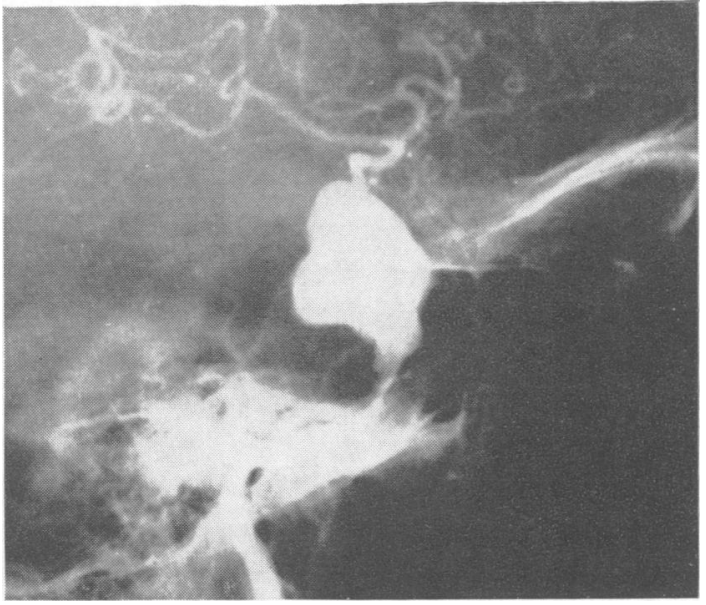

FIG. 5

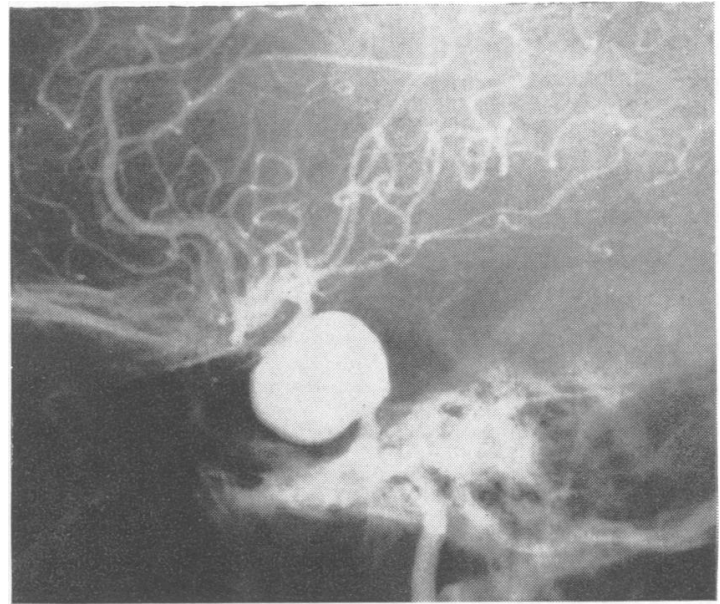

FIG. 6

FIG. 1. Photograph of patient, showing partial ophthalmoplegia of the right eye.

FIG. 2. Plain skull film, antero-posterior projection, showing erosion of the right superior orbital fissure.

FIG. 3. Right carotid arteriogram, antero-posterior projection, shows stretching of the middle cerebral artery over the aneurysm.

FIG. 4. Left carotid arteriogram, antero-posterior view, shows cross filling during occlusion by manual compression of the right internal carotid artery.

FIG. 5. Right carotid arteriogram, lateral view, shows intracavernous saccular aneurysm, poor filling of the anterior cerebral artery, elevation of the internal carotid artery bifurcation, and kinking of the internal carotid artery proximal to the bifurcation.

FIG. 6. Left carotid arteriogram, lateral view, demonstrates the left intracavernous aneurysm. 
TABLE I

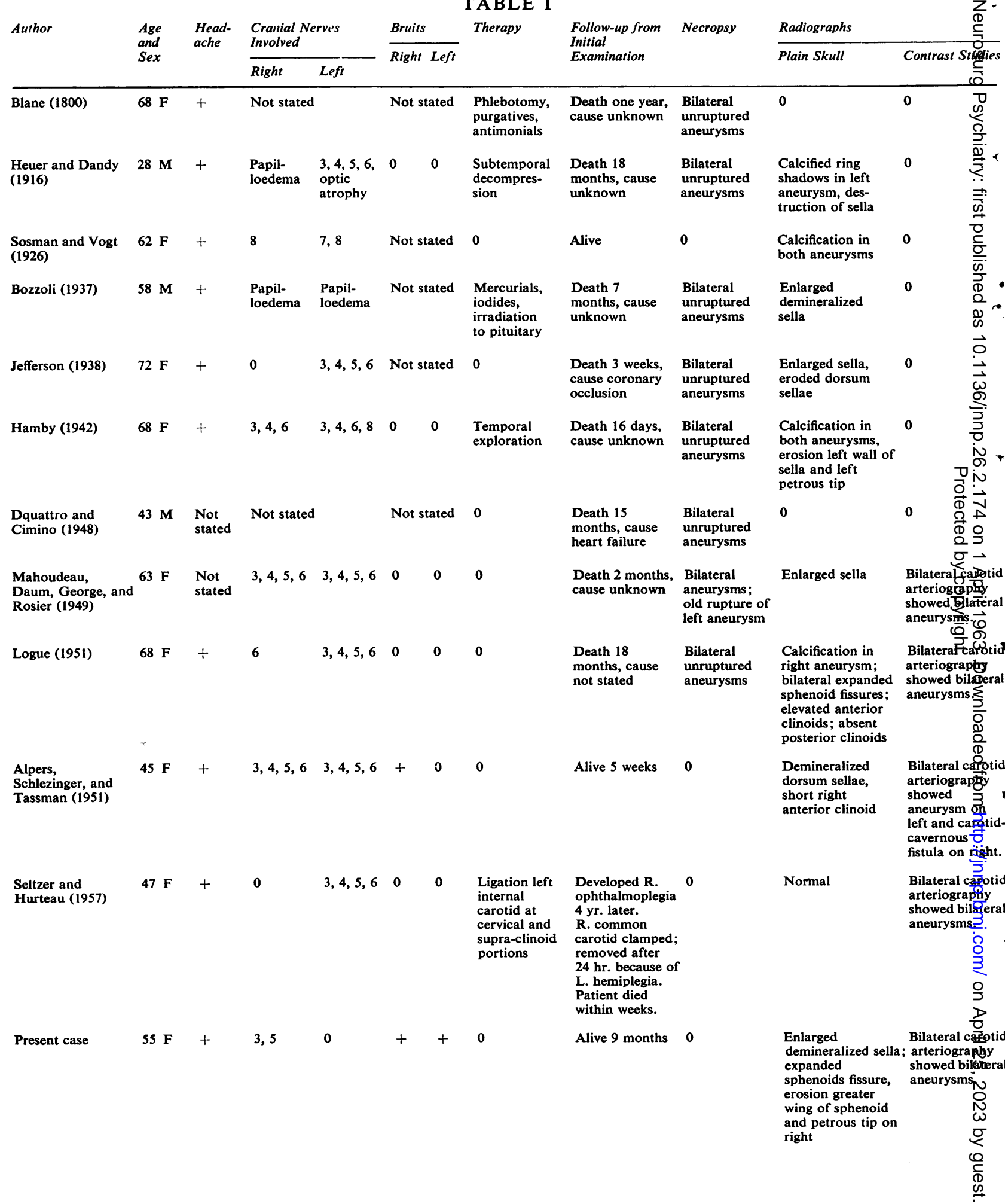




\section{DISCUSSION}

The cause for convulsions in the post-angiography period remained obscure. The angiogram was done with local anaesthesia. Vascular spasm seemed unlikely because at no time was there any evidence of subarachnoid haemorrhage. Convulsions may occur immediately after injection of the angiographic medium but usually this is a complication in patients who have a history of convulsions or have demonstrable cerebral pathology. Neither of these factors applied in the present case, and the temporal relationship of the procedure to the convulsions made a direct irritative effect of the contrast medium unlikely.

The therapeutic problem created by the presence of bilateral aneurysms deserves careful consideration. The decision regarding any surgical procedure must take three factors into account: the risk of the procedure itself, the course of the untreated disease, and the benefit to be realized by the patient from the contemplated procedure.

The only patient with bilateral aneurysms on whom a surgical procedure has been carried out was that reported by Seltzer and Hurteau (1957). In their case, the indications for operative intervention were impairment of ocular motility, blurred vision, and throbbing headache. Orbital pain, the most frequent symptom requiring treatment, was not mentioned. The left internal carotid artery was occluded proximal and distal to the symptomatic aneurysm. Eye movements were restored but fine movements in the right hand were impaired. This patient (Hurteau, 1962) was readmitted four years later with complete ophthalmoplegia on the right side. The right common carotid artery was gradually occluded with a Selverstone clamp, which was opened 24 hours after its application after the sudden development of left hemiplegia. There was slight improvement in the external ophthalmoplegia; however, the hemiplegia persisted. The patient died in a convalescent home several weeks later. No necropsy was obtained.

The morbidity and mortality of cervical carotid ligation in our experience has been unpredictable, and gradual occlusion with a clamp has not entirely avoided these complications. The deleterious effect on the smaller and asymptomatic aneurysm of ligating the contralateral carotid artery cannot be discounted. Secondly, the course of the untreated disease does not seem immediately or necessarily unfavourable, because intracavernous aneurysms may pursue a protracted course. Finally, the necessity for relieving the patient's complaints should be questioned. The right retro-orbital pain in our patient was mild in nature and intermittent, in contrast to the 17 patients reported by Jefferson (1938), all of whom had intense pain. It was realized that pain might become a problem in the future, but this possibility did not constitute an indication for prophylactic carotid ligation. The smaller asymptomatic aneurysm could also produce pain in the future, either with or without ligation of the contralateral vessel. If incapacitating pain develops, carotid ligation will be considered.

\section{SUMMARY}

A case of bilateral intracavernous carotid aneurysms diagnosed by carotid arteriography is presented. Eleven cases have been found in the literature to date, four of which were diagnosed during life. A tabular summary of all cases is given.

\section{REFERENCES}

Alpers, B. J., Schlezinger, N. S., and Tassman, I. M. (1951). A.M.A. Arch. Ophthal., 46, 403.

Blane, G. (1800). Trans. Soc. Improvement med. chir. Knowledge, 2 , 192.

Bozzoli, A. (1937). Riv. oto-neuro-oftal., 14, 304.

Dquattro, C., and Cimino, G. (1948). Pathologica, 40, 111.

Hamby, W. B. (1942). J. int. Coll. Surg., 5, 216. Heuer, G. J., and Dandy, W. E. (1916). Bull. Johns Hopk. Hosp., 27,

Hurteau, E. F. (1962). Personal communication.

Jefferson, G. (1938). Brit. J. Surg., 26, 267.

Logue, V. (1951). Ibid., 39, 181.

Mahoudeau, D., Daum, S., George, and Rosier (1949). Bull. Soc. méd. Hôp. Paris, 65, 503.

Rischbieth, R. H. C., and Bull, J. W. D. (1958). Brit. J. Radiol., 31, 125.

Seltzer, J., and Hurteau, E. F. (1957). J. Neurosurg., 14, 448

Sosman, M. C., and Vogt, E. C. (1926). Amer. J. Roentgenol., 15, 122. 